\title{
Efectos de la imposición de aranceles a productos agropecuarios de Estados Unidos en importaciones de México, TLCAN
}

\section{Yuselim Angélica Villa Hernández*, Antonto Kido Cruz** Virginia Hernández Silva** y Salvador Madrigal Moreno**}

\section{RESUMEN}

En el marco de las renegociaciones del Tratado de Libre Comercio de América del Norte (TLCAN), y derivado de la imposición de aranceles al acero y al aluminio de Estados Unidos a todos los países, la respuesta de México ha sido gravar diversos productos agropecuarios de Estados Unidos, lo cual podría originar aumentos de precios a nivel nacional. En el presente estudio se determinan y analizan, mediante la Teoría Estructural y el Modelo de Precios, los efectos multiplicadores que, derivado de lo anterior, se generarán dentro de los sectores agropecuario, industrial y de servicios en México. Se confirma que los impuestos arancelarios a la importación de los productos agropecuarios generan un incremento de precios en los tres sectores económicos del país, por ello México debe encontrar una alternativa a la negociación del TLCAN que beneficie a los tres involucrados.

Palabras clave: Sector agropecuario, TLCAN, Teoría del Análisis Estructural, Modelo de Precios.

Clasificación JEL: F51, Q17

Facultad de Contaduría y Ciencias Administrativas, Universidad Michoacana de San Nicolás de Hidalgo, México. Correo electrónico: yuselim.23@gmail.com.

** Profesores investigadores de tiempo completo, Universidad Michoacana de San Nicolás de Hidalgo, México. Correo electrónico: akido42@hotmail.com, vhsilva_17@hotmail.com y smadrigal@umich.mx, respectivamente. 


\section{ABSTRACT}

\section{Effects of the imposition of tariffs to the agricultural products of the United States in the imports of Mexico, NAFTA}

In the framework of the renegotiations of the North American Free Trade Agreement (NAFTA) and derived from the imposition of tariffs on steel and aluminum from the United States to all countries, including Mexico, Mexico's retaliation has been to impose tariffs on agricultural products to the United States. In the present study, the multiplier effects between the price increases in the imports of the agricultural sector of Mexico and the agricultural, industrial and service sectors are determined and analyzed through the Structural Theory and the Price Model. It is confirmed that the tariff taxes on the importation of agricultural products have a negative effect on the three economic sectors of the country. That is why Mexico must find another solution to the NAFTA negotiation that benefits the three countries involved.

Key words: Agricultural sector, NAFTA, Theory of Structural Analysis, Price Model.

\section{JEL classification: F51, Q17}

\section{INTRODUCCIÓN}

Debido a la globalización, en las últimas décadas el comercio internacional ha sido regulado por diversos acuerdos como el Tratado de Libre Comercio de América del Norte (TLCAN), que establece las normas arancelarias entre Estados Unidos, México y Canadá. Durante este año se ha estado renegociando con miras a generar beneficios para las tres partes involucradas; ante la importancia de que se conozcan los alcances económicos de los reajustes, el propósito del presente trabajo de investigación radica en llegar a una aproximación de los efectos multiplicadores de los aranceles impuestos a los productos agropecuarios en la economía nacional, con impacto directo en el sector agropecuario por el aumento de precios en las importaciones e indirecto en el de servicios e industrial.

La Matriz Insumo Producto (MIP) más reciente que elabora y publica el Instituto Nacional de Geografía y Estadística (INEGI) es la fuente de información de esta investigación; se parte de un análisis de la 
MIP desde el enfoque de la Teoría del Análisis Estructural y se observa que, efectivamente, los tres sectores de la economía guardan interdependencia; de manera empírica se calculan sus efectos multiplicadores mediante álgebra matricial y considerando la Matriz de Leontief. Dado que la economía de México resulta abierta, se estima el efecto multiplicador del aumento de costos de importaciones del sector agropecuario en los precios. Finalmente, se llega a las conclusiones derivadas de los resultados de la metodología empírica.

\section{México y el TRATAdo de Libre Comercio de América DEL NORTE}

Desde la perspectiva de México, según Appendini (2018), el TLCAN fue un proyecto político, pero también económico, puesto en marcha por el Estado mexicano con el objeto de establecer reformas económicas liberales; es decir, fue basado en la premisa del libre comercio con beneficios para las partes involucradas.

La renegociación del TLCAN conlleva un clima de incertidumbre respecto a las decisiones de los países miembros; en caso de que Estados Unidos abandonara el TLCAN, estarían vigentes las normas comerciales de la Organización Mundial de Comercio (OMC). En este contexto, entrarían en vigor los aranceles de la Nación Más Favorecida que, aunque son relativamente bajos para los productos agropecuarios, pueden impactar en toda la economía (Gazcón, 2017).

\subsection{El sector agroalimentario en México y el TLCAN}

La economía mexicana ha promovido su crecimiento principalmente por las labores terciarias, mientras que ha relegado las primarias, si bien éstas continúan siendo muy importantes para el desarrollo de otros sectores, en tanto impulsoras de la actividad económica.

Por ello, Urrutia et al. (2017) se enfocan al análisis de la contribución del sector pecuario a la economía, con énfasis en los aportes a la producción nacional y su relevancia en la demanda intermedia, mediante la MIP. Ello permitió conocer la oferta y demanda intersectorial e identificar los sectores más importantes en la economía; determinaron que los principales encadenamientos productivos de las actividades pecuarias se encuentran en el interior, con una demanda de insumos de 69\% para 
los sistemas ovino-caprino, $52 \%$ para el bovino, $46 \%$ para el porcino y el 45\% para el avícola.

Yúnez y González (2008), en su estudio sobre los efectos multiplicadores de las actividades productivas en ingreso y pobreza rural en México, lograron estimar, a partir de un enfoque multisectorial, las consecuencias por cambios en las actividades productivas. Para ello, elaboraron una matriz de contabilidad social agregada de diez comunidades rurales mexicanas caracterizadas por la pobreza predominante en sus hogares; aplicaron la técnica de descomposición de multiplicadores. Los resultados muestran que dentro de las cinco actividades productivas de los hogares rurales, la agricultura comercial posee los efectos multiplicadores más altos en el ingreso y en la disminución de la pobreza; por lo tanto, destaca la conveniencia de que el Estado apoye la producción de cultivos para su comercialización a partir, por ejemplo, de inversiones en infraestructura pública (comunicaciones y transporte).

De acuerdo con Celis (2018), en 2017 las exportaciones mexicanas en el sector agroalimentario (principalmente hortalizas y frutas frescas) ascendieron a 32583 millones de dólares, lo que llevó a un aumento de $70 \%$ del superávit de la balanza comercial respecto al de 2016; de acuerdo con lo que informó José Calzada, el secretario de Agricultura, $80 \%$ de las ventas se dirigió a Estados Unidos.

Los granos forrajeros, oleaginosas y productos de origen animal constituyen las principales importaciones a México de Estados Unidos. La Secretaría de Economía publicó, en el Diario Oficial de la Federación del 5 de junio de 2018, el decreto por el que impuso un arancel de $20 \%$ a las importaciones de carne de cerdo, manzanas, arándanos y papas estadounidenses, así como tarifas de entre 20 y $25 \%$ sobre varios tipos de quesos (DOF, 2018), acciones derivadas de la imposición de aranceles al acero y aluminio por el gobierno de Donald Trump.

Ramos (2017) señala que el mercado de hortalizas y frutas frescas en Estados Unidos se ha ido consolidando a través de los años; si el TLCAN se cancela, los consumidores tendrían que pagar un precio superior por los productos de importación adquiridos, pues el arancel sería de $7 \%$.

Asimismo, muchas de las hortalizas exportadas al país del norte emplean insumos, bienes y servicios producidos por empresas trasna- 
cionales de origen estadounidense. La cancelación del TLCAN podría disminuir dramáticamente las ventas de esas mercancías en México, en perjuicio de su desarrollo y crecimiento.

Al respecto, el secretario de Economía del Gobierno de Enrique Peña Nieto, Ildefonso Guajardo, aseguró, en el V Encuentro Empresarial de la Alianza del Pacífico, que sí hay una ventana de oportunidad para llegar a un acuerdo en las negociaciones del TLCAN en agosto de 2018, pero en forma bilateral porque el representante comercial de Estados Unidos, Robert Lighthizer, negociará por separado, primero con México y luego con Canadá.

Tanto Guajardo como Jesús Seade, el coordinador designado en el equipo de transición, han insistido en mantener la trilateralidad del TLCAN. Guajardo ha explicado al respecto que, para firmar un acuerdo bilateral, Estados Unidos tendría primero que renunciar al TLCAN y solicitar la autorización de su Congreso, lo que resulta sumamente complicado en el actual entorno político de Estados Unidos (Cortés, 2018).

Por otra parte, Carbajal y Del Moral (2014) sostienen que uno de los mecanismos más importantes del comercio internacional se basa en los procesos de integración económica entre dos o más naciones, mediante los cuales los países van eliminando las disposiciones que otorgan tratamientos diferenciales entre ellos - es decir, medidas arancelarias y no arancelarias o desplazamiento e intercambio de mercancías de una frontera a otra- a través de acuerdos internacionales, cuya finalidad es reducir o anular el cobro de restricciones arancelarias para propiciar un mayor tránsito, no sólo de mercancías y servicios, sino de inversiones directas y de tecnología. Lo contrario a la integración económica es el fenómeno generado entre los integrantes del TLCAN, a partir de la desventaja en que se coloca a México con el aumento de aranceles en aluminio y acero.

Con base en la premisa de que, en el marco del libre comercio y en un mercado perfecto, un bien se debe vender al mismo precio en dos países, Yúnez y Barceinas (2004) efectuaron un análisis econométrico para estudiar los cambios estructurales de la relación entre los precios nacionales y extranjeros de los principales productos agrícolas a partir del TLCAN, con el propósito de estudiar la convergencia entre México y Estados Unidos. Concluyeron que, durante los últimos 22 
años, el precio interno de los cultivos estudiados ha tendido a seguir a Estados Unidos, mientras que los de algunas de las hortalizas y frutas exportadas más importantes se han vinculado en mayor medida con los costos internacionales; en definitiva, el comercio agrícola en general ha crecido durante la implementación del TLCAN.

Camargo (2017) expresa que, si bien el desarrollo productivo de México depende de la capacidad de los factores de producción para aprovechar las oportunidades generadas por la liberación y apertura económicas, también implica la renovación de políticas y estrategias que favorezcan un proceso gradual de equilibrio entre los mercados. La renegociación del TLCAN debe proveer las condiciones idóneas para el crecimiento equitativo de todas las regiones y sectores económicos del país.

\section{TEORÍA DEL ANÁLISIS ESTRUCTURAL}

De acuerdo con Leontief (1993), el Modelo Insumo-Producto constituye una adaptación de la teoría neoclásica del equilibrio al estudio de la interdependencia cuantitativa existente entre actividades económicas que mantienen una relación recíproca.

La producción de cada sector está destinada a venderse en el mercado como producto intermedio o final; en el primer caso, se emplea durante el último proceso productivo; en el segundo, se comercializa entre los residentes de un país como bien de consumo o inversión. Cuando se destina a los no residentes tienen lugar las exportaciones, por lo que el valor de la producción del sector i-ésimo puede denotarse en forma matemática en la siguiente expresión:

Donde:

$$
x_{i}=z_{i 1}+\cdots+z_{i j}+\cdots+z_{i n}+f_{i}
$$

$\mathrm{Xi}=$ valor de la producción del sector i-ésimo

$\mathrm{Zij}=$ valor de las ventas interindustrias del sector i-ésimo, que vende a todos los sectores $\mathrm{j}$ incluyéndose a sí mismo, cuando $\mathrm{j}=\mathrm{i}$

$\mathrm{fi}=$ valor de la demanda final, integrado por consumidor doméstico, inversión, gobierno y exportación

Supuestos:

1) El funcionamiento de una economía se traduce en el valor de la producción de " $n$ " sectores. 
2) La producción del sector i-ésimo es igual a xi

3) La función de producción es fija y se expresa como:

$$
\mathrm{xj}=\mathbf{x i j} / \mathbf{a i j}
$$

4) Las variables endógenas son las $n+n 2$ siguientes:

$$
\text { xi con } i=1,2, \ldots \text { n y } x i j \operatorname{con} i j=1,2, \ldots n
$$

5) Las variables exógenas son Consumidor doméstico, inversión, gobierno, exportaciones.

El procedimiento establecido por Leontief consiste en determinar la interrelación de los diversos sectores de la estructura abordada por medio de ecuaciones lineales, cuyos coeficientes numéricos simbolizan las particularidades del sistema en sí mismo. De forma empírica se obtienen los valores de estos coeficientes: si aluden a la economía de una nación, normalmente se consiguen mediante una tabla estadística Insumo-Producto; este análisis constituye una herramienta de gran valor para planear la producción económica de los países. La relevancia del presente trabajo se justifica con la propuesta de Burgos (2007), quien señala las diversas aplicaciones de la MIP: decisiones empresariales, proyecciones de comercio exterior, así como análisis de precios y costos.

En estudios anteriores, como el desarrollado en el estado de Baja California por Fuentes (2005), se estima la MIP desde el enfoque indirecto conocido como partial survey method, basado en el procedimiento tipo RAS, pero ajustado al espacio y a la información exógena disponible de las transacciones intersectoriales. El propósito de estimar la MIP de manera regional fue detallar el proceso de generación de datos locales $\mathrm{y}$, además, promover la participación activa de los interesados en la discusión del análisis de la zona.

En el nivel empírico, el uso y la aplicación de la MIP facilita el conocimiento y la comprensión del mundo de las relaciones intersectoriales, además del nivel de integración de una economía regional como un todo. Por lo tanto, representa un instrumento útil para la identificación de actividades económicas relevantes desde el punto de vista de los efectos multiplicadores del gasto, del empleo y de la producción y, en consecuencia, una guía para el diseño de políticas públicas y la toma de decisiones en la asignación de recursos de inversión. 


\subsection{Teoría del Modelo de Precios}

Es bien conocido que la versión dual del Modelo regular de InsumoProducto se aplica en la simulación de procesos inflacionarios de empuje de costos; por supuesto, esta versión de doble precio del Modelo de Leontief se basa en los mismos supuestos estrictos y estándares de la cuantitativa y en algunas suposiciones adicionales para la de precio (Oosterhaven, 1996: 750). En la versión cuantitativa del Modelo de Leontief, la expresión matemática que sustenta al desarrollo del modelo considera los elementos de la MIP expresados en el renglón, es decir, los productos demandados por los sectores del conjunto económico. Además, se incluye un vector de demandas finales $f_{i} \circ y_{i}$, el cual origina el empuje del modelo, razón de que usualmente se denomine Modelo de Empuje por el Lado de la Demanda, a diferencia del Modelo de Precios (Chávez, 2017:6).

El estudio de la variación en la producción, originada por el cambio de una variable exógena (como la demanda final), se realiza a través de la versión cuantitativa del Modelo de Leontief, o Modelo de Empuje por el Lado de la Demanda (Oosterhaven, 1996: 750), en cuyo análisis se asume el supuesto de coeficientes técnicos constantes.

El Modelo de Precios permite conocer las variaciones de los distintos bienes producidos en la economía, como resultado de un incremento en los costos de los insumos primarios (Dietzenbacher, 1997:631), por ello, también se le denomina Modelo de Empuje por el Lado de Costos.

\section{MATERIALES Y MÉTODOS}

\subsection{MIP general de México, generada por el INEGI}

La MIP general de México (se considera 2013 como el año base) servirá como ejemplo para explicar la conformación de una matriz de tal naturaleza; las cantidades se expresan en millones de pesos a precios básicos de 2013 . 


\section{Cuadro 1}

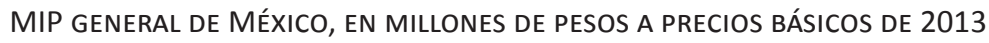

\begin{tabular}{cccccc}
\hline & \multicolumn{3}{c}{ DEMANDA INTERMEDIA } & & \\
\cline { 2 - 4 } ACTIVIDAD & $\begin{array}{c}\text { Sector } \\
\text { primario }\end{array}$ & $\begin{array}{c}\text { Sector } \\
\text { industrial }\end{array}$ & $\begin{array}{c}\text { Sector de } \\
\text { servicios }\end{array}$ & $\begin{array}{c}\text { DEMANDA } \\
\text { FINAL }\end{array}$ & $\begin{array}{c}\text { PRODUCCIÓN } \\
\text { TOTAL }\end{array}$ \\
Sector primario & 64,515 & 421,732 & 5,170 & 288,326 & 779,742 \\
\hline Sector industrial & 117,098 & $3,021,320$ & 746,758 & $9,753,927$ & $13,639,102$ \\
\hline Sector de servicios & 55,740 & $1,853,896$ & $1,805,456$ & $9,508,712$ & $13,223,804$ \\
\hline Actividades industriales & 237,353 & $5,296,948$ & $2,557,383$ & $19,550,963$ & $27,642,648$ \\
\hline Importaciones & 65,594 & $3,200,166$ & 633,124 & $1,386,205$ & $5,283,449$ \\
\hline Impuestos & -419 & $-19,679$ & 29,556 & 625,109 & 634,567 \\
\hline Total de usos a precios de & 302,529 & $8,477,436$ & $3,220,064$ & & $33,560,664$ \\
comprador & & & & & $15,642,620$ \\
\hline Valor agregado bruto & 477,213 & $5,161,666$ & $10,003,740$ & & $27,642,648$ \\
\hline Producción & 779,742 & $13,639,102$ & $13,223,804$ & & $16,277,187$ \\
\hline PIB & 476,794 & $5,141,987$ & $10,033,297$ & & \\
\hline
\end{tabular}

Fuente: Elaboración propia con base en la MIP de México a precios básicos de 2013 (INEGI, 2017).

De acuerdo con Miller y Blair (2009), un sistema Insumo-Producto consta de una estructura conformada por un conjunto de $\mathrm{n}$ ecuaciones lineales con $\mathrm{n}$ incógnitas, lo cual facilita el empleo de las representaciones matriciales. Se elabora partiendo de datos observados en una particular área económica (un estado, una región, una nación, entre otras); según estos autores, en un principio se supondrá que esta área económica corresponde a un país, cuya economía puede ser separada en segmentos o sectores productivos.

El equilibrio sectorial entre la oferta y la utilización de los bienes y servicios de una economía se expresa, en forma matricial, mediante una MIP: explica de manera concisa la economía de una región; asimismo, facilita el análisis y la evaluación, de acuerdo con ciertas presunciones de tecnología, de las necesidades de producción de cada sector que satisfagan los requerimientos de consumo e inversión, a fin de determinar 
el nivel de producción originado por un incremento en la demanda (Hernández, 2012).

Para entender la aplicación del análisis Insumo-Producto, en primer lugar, se parte de que este modelo divide la economía de un país en $\mathrm{n}$ sectores. La producción bruta efectiva se define con $\mathrm{x}_{\mathrm{ny}}$, debido a que el método de Leontief implica un estudio de la circulación de insumos y productos entre sectores, éstos se expresan como $z_{i j}$; de igual forma, $z_{i j}$ manifiesta la cantidad de producto del sector $i$ consumida por el sector $j$. En segundo lugar, resulta esencial comprender que no todo el producto de i será destinado al resto de los sectores, dado que una parte se emplea en la demanda final (Chraki, 2016: 58).

Hay una ecuación que identifica las ventas de los productos de cada uno de los sectores $\boldsymbol{n}$ :

$$
\begin{aligned}
& x_{1}=z_{11}+\cdots+z_{1 j}+\cdots+z_{1 n}+f_{1} \\
& x_{i}=z_{i 1}+\cdots+z_{i j}+\cdots+z_{i n}+f_{i} \\
& x_{n}=z_{n 1}+\cdots+z_{n j}+\cdots+z_{n n}+f_{n}
\end{aligned}
$$

Donde:

$$
\begin{gathered}
\mathrm{X}=\left[\begin{array}{c}
x_{1} \\
\vdots \\
x_{n}
\end{array}\right] \\
\begin{array}{c}
\text { Producción total } \\
\text { de un sector }
\end{array}
\end{gathered}
$$$$
Z=\left[\begin{array}{ccc}
Z_{11} & \cdots & Z_{1 n} \\
\vdots & \ddots & \vdots \\
Z_{n 1} & \cdots & Z_{n n}
\end{array}\right]
$$$$
f=\left[\begin{array}{c}
f_{1} \\
\vdots \\
f_{n}
\end{array}\right]
$$$$
\text { Demanda intermedla }
$$

Demanda final del sector

La distribución de las ventas de cada sector puede resumirse con la siguiente notación matricial:

$$
x=Z i+f
$$

Leontief (1993) explica, con objeto de facilitar la manipulación matemática, que la producción física total de un sector i se puede representar como $x_{i}$, y la cantidad de producto de $\mathrm{i}$ absorbida — en calidad de insumo- - por el sector $\mathrm{j}$ por $\mathrm{z}_{\mathrm{ij}}$. La cantidad de producto de $\mathrm{i}$ que pasa al sector que representa la demanda final $\mathrm{z}_{\mathrm{i}, \mathrm{n}+1}$ suele expresarse como $\mathrm{y}_{\mathrm{i}}$.

Ahora, mediante el desarrollo matemático de Miller y Blair (2009), se presenta una tabla Insumo-Producto para una pequeña economía de 
tres sectores; al aplicar la siguiente ecuación, en la cual se alude a la demanda final como $\mathrm{y}_{\mathrm{i}}$, se tiene:

$$
\begin{aligned}
& x_{1}=z_{11}+z_{12}+z_{13}+y_{1} \\
& x_{2}=z_{21}+z_{22}+z_{23}+y_{2} \\
& x_{3}=z_{31}+z_{32}+z_{33}+y_{3}
\end{aligned}
$$

En donde la producción total para cada sector está dada por $\mathrm{x}_{1}, \mathrm{x}_{2} \mathrm{y} \mathrm{x}_{3}$; la demanda intermedia, por cada interrelación $\mathrm{z}_{\mathrm{ij}}$ (por ejemplo, $\mathrm{z}_{11}$ es la producción del sector 1 consumida o utilizada en el sector 1 , y así sucesivamente); y la demanda final, por $\mathrm{y}_{1}, \mathrm{y}_{2} \mathrm{y}_{3}$.

En las ecuaciones expresadas, las columnas indican la estructura de costos de cada sector $\mathbf{y}$, si se representa mediante el símbolo $\mathrm{a}_{\mathrm{ij}}$ la cantidad de producto del sector $\mathbf{i}$ consumida por cada sector $\mathrm{j}$ por unidad de su producción total $\mathbf{j}$ - lo que equivale a dividir el valor de cada insumo entre el valor bruto de producción correspondiente (el total de la columna)—, se obtienen los denominados coeficientes de insumo o coeficientes técnicos.

Los coeficientes técnicos registran la necesidad de insumos del sector $\mathbf{i}$ para producir una unidad del producto en el sector $\mathbf{j}$; está dada por la siguiente expresión:

$$
a_{i j}=\frac{z_{i j}}{x_{j}}
$$

Donde $\mathrm{i}$ indica el sector que vende $\mathrm{y} \mathrm{j}$, el sector que produce; entonces, despejando Z11, Z12, y así sucesivamente, se tiene:

$$
\begin{aligned}
& x_{1} a_{11}=z_{11} \\
& x_{2} a_{12}=z_{12} \\
& x_{3} a_{13}=z_{13}
\end{aligned}
$$

Una vez que el concepto de conjunto de coeficientes técnicos es aceptado, las ecuaciones de (5) se pueden reescribir (sustituyendo cada $\mathrm{z}_{\mathrm{ij}}$ por $\mathrm{x}_{\mathrm{j}}$ aij) como:

$$
\begin{aligned}
& x_{1}=x_{1} a_{11}+x_{2} a_{12}+x_{3} a_{13}+y_{1} \\
& x_{2}=x_{1} a_{21}+x_{2} a_{22}+x_{3} a_{23}+y_{2} \\
& x_{3}=x_{1} a_{31}+x_{2} a_{32}+x_{3} a_{33}+y_{3}
\end{aligned}
$$


Así, la demanda final se obtiene despejando y de cada ecuación:

$$
\begin{aligned}
& x_{1}-x_{1} a_{11}-x_{2} a_{12}-x_{3} a_{13}=y_{1} \\
& x_{2}-x_{1} a_{21}-x_{2} a_{22}-x_{3} a_{23}=y_{2} \\
& x_{3}-x_{1} a_{31}-x_{2} a_{32}-x_{3} a_{33}=y_{3}
\end{aligned}
$$

Ahora, factorizando $\mathrm{x} 1$ en la primera ecuación, $\mathrm{x} 2$ en la segunda y x3 en la tercera, se tiene:

$$
\begin{aligned}
(1- & \left.a_{11}\right) x_{1}-x_{2} a_{12}-x_{3} a_{13}=y_{1} \\
& -x_{1} a_{21}+\left(1-a_{22}\right) x_{2}-x_{3} a_{23}=y_{2} \\
& -x_{1} a_{31}-x_{2} a_{32}+\left(1-a_{33}\right) x_{3}=y_{3}
\end{aligned}
$$

De este modo se expresa en forma matricial la anterior ecuación:

$$
C=\left[\begin{array}{ccc}
\left(1-a_{11}\right) & -a_{12} & -a_{13} \\
-a_{21} & \left(1-a_{22}\right) & -a_{23} \\
-a_{31} & -a_{32} & \left(1-a_{33}\right)
\end{array}\right] x=\left[\begin{array}{l}
x_{1} \\
x_{2} \\
x_{3}
\end{array}\right] y=\left[\begin{array}{l}
y_{1} \\
y_{2} \\
y_{3}
\end{array}\right]
$$

Estas relaciones se pueden representar de manera compacta en forma de matriz. En álgebra matricial, el símbolo ^ sobre un vector expresa una matriz diagonal con los elementos del vector a lo largo de la diagonal principal, por ejemplo:

$$
\text { El vector } x=\left[\begin{array}{c}
x_{1} \\
\vdots \\
x_{n}
\end{array}\right] \text { se expresa como } \hat{x}=\left[\begin{array}{ccc}
x_{1} & \cdots & 0 \\
\vdots & \ddots & \vdots \\
0 & \cdots & x_{n}
\end{array}\right]
$$

Ahora, de la definición básica de matriz inversa, $\left((\mathrm{x})^{\wedge}\right)(\mathrm{x})^{\wedge}(-1)=\mathrm{I}$, por lo que resulta que:

$$
\hat{x}^{-1}=\left[\begin{array}{ccc}
1 / x_{1} & \cdots & 0 \\
\vdots & \ddots & \vdots \\
0 & \cdots & 1 / x_{n}
\end{array}\right]
$$

La postmultiplicación de una matriz M por una matriz diagonal d̂ crea una matriz en la cual cada elemento en la columna j de $\mathrm{M}$ es multiplicada por $\mathrm{d}_{\mathrm{j}}$ en $\hat{\mathrm{d}}$, por lo tanto, la matriz $\mathrm{n} \times \mathrm{n}$ de coeficientes técnicos puede expresarse como: 


$$
A=Z \hat{x}^{-1}
$$

La expresión matricial se manifiesta del siguiente modo:

$$
x=A x+y
$$

Ahora, de las matrices asentadas se tiene que:

$$
C x=y
$$

Si se descompone $\mathrm{C}$ en la resta de dos matrices, se obtiene:

$$
I=\left[\begin{array}{lll}
1 & 0 & 0 \\
0 & 1 & 0 \\
0 & 0 & 1
\end{array}\right] \text { y } A=\left[\begin{array}{lll}
a_{11} & a_{12} & a_{13} \\
a_{21} & a_{22} & a_{23} \\
a_{31} & a_{32} & a_{33}
\end{array}\right]
$$

La ecuación expresada queda:

$$
(I-A) x=y
$$

Donde x (producción total de cada sector) es la variable dependiente, mientras y es la independiente (demanda final correspondiente a cada sector), por lo que se requiere encontrar una matriz (I-A) ${ }^{-1}$ inversa que multiplicada por (I-A) de la unidad (identidad):

$$
(I-A)^{-1}(I-A) x=(I-A)^{-1} y
$$

Entonces:

$$
I x=(I-A)^{-1} y
$$

Por lo tanto:

$$
x=(I-A)^{-1} y
$$

Donde $(\mathrm{I}-\mathrm{A})^{-1}=\mathrm{L}$ es la conocida inversa de Leontief, que se puede expresar como:

$$
x=L y
$$

Donde $\mathrm{x}$ es la producción total de cada sector; L, la matriz inversa de la matriz A de coeficientes técnicos; $y$, la demanda final correspondiente a cada sector. 


\subsection{Efectos multiplicadores}

En concordancia con Ramos et al. (20013), se identifican como efectos multiplicadores aquellos cambios originados por alteraciones en una variable exógena — por ejemplo, la demanda final—, en un sector de la economía de un país.

El estudio de los efectos directos y multiplicadores de un sector específico sobre el resto de los que conforman un sistema económico se realiza a través de la matriz de coeficientes de necesidades directas o coeficientes técnicos. Esta matriz, el núcleo del estudio, explica la vinculación inmediata entre sectores; se calcula mediante la división de los elementos de la demanda intermedia de cada sector por su valor de producción correspondiente (Figueroa y Obed, 2015).

El Cuadro 2, resultado de aplicar la metodología de Núñez y Romero (2016), muestra que, si se llegara a presentar un aumento en la demanda final de uno de los tres sectores indicados, la producción correspondiente tendría que incrementarse; por consiguiente, la demanda de insumos se elevaría y ocasionaría el crecimiento de la producción de los demás sectores; la suma de todos estos efectos se conoce como efecto multiplicador total.

Cuadro 2

MATRIZ DE COEFICIENTES TÉCNICOS PARA LA MIP 2013 DOMÉSTICA POR TIPO DE ACTIVIDAD

\begin{tabular}{cccc}
\hline \multirow{2}{*}{ Actividades } & \multicolumn{3}{c}{ Matriz de Coeficientes Técnicos } \\
& Actividades primarias & Actividades industriales & Actividades de servicios \\
Actividades primarias & 0.0827 & 0.0309 & 0.0004 \\
\hline Actividades industriales & 0.1502 & 0.2215 & 0.0565 \\
\hline Actividades de servicios & 0.0715 & 0.1359 & 0.1365 \\
\hline Importaciones & 0.0841 & 0.2346 & 0.0479 \\
Impuestos sobre los & & & 0.0022 \\
productos netos de & -0.0005 & -0.0014 & \\
subsidios & & & 0.7565 \\
\hline Valor agregado bruto & 0.6120 & 0.3784 & 1.0000 \\
\hline Producción & 1.0000 & 1.0000 & \\
\hline
\end{tabular}

Fuente: Elaboración propia con base en la MIP de México a precios básicos de 2013 (INEGI, 2017). 


\section{3. Índices de Rasmussen}

Dentro de los métodos existentes para identificar los sectores claves de una economía, esencialmente los enfocados al peso de cada uno para generar efectos de arrastre (tanto hacia adelante como hacia atrás, sin importar su tamaño), se cuenta con los denominados índices de Rasmussen, cuya aplicación resulta extensa dado que proporcionan un primer acercamiento apropiado al análisis de la estructura de una economía real (Núñez y Romero, 2016). La capacidad de originar impactos de arrastre hacia atrás corresponde a la magnitud que un incremento presentado en el sector j origina en los demás, mientras que el caso opuesto se identifica como la magnitud en que el sistema económico pesa sobre la industria i, es decir, la medida en que i es afectada por un aumento en el sistema económico. Los cálculos del índice de Rasmussen se presentan en el Cuadro 3.

Cuadro 3

ÍNDICES DE RASMUSSEN PARA LA MIP 2013 DOMÉSTICA POR TIPO DE ACTIVIDAD

\begin{tabular}{cccccc}
\hline Actividades & $\mathbf{1}$ & $\mathbf{2}$ & $\mathbf{3}$ & $\mathbf{f}_{\mathbf{1}}$ & Producción total \\
\hline 1 & 64,515 & 421,732 & 5,170 & 288,325 & 779,742 \\
\hline 2 & 117,098 & $3,021,320$ & 746,758 & $9,753,926$ & $13,639,102$ \\
\hline 3 & 55,740 & $1,853,896$ & $1,805,456$ & $9,508,711$ & $13,223,804$ \\
\hline Fuerza laboral & 542,389 & $8,342,154$ & $10,666,420$ & $2,009,673$ & $21,560,636$ \\
\hline Producción & 779,742 & $13,639,102$ & $13,223,804$ & $21,560,636$ & \\
\hline
\end{tabular}

Fuente: Elaboración propia con base en la MIP de México a precios básicos de 2013 (INEGI, 2017).

Se aprecia que el sector agropecuario no es de dispersión en tanto el coeficiente estimado resulta menor a la unidad, pero sí se caracteriza como uno de arrastre por el coeficiente de 1.053; en otras palabras, el sector agropecuario en México representa más un sector impulsor de la economía que uno estratégico.

\section{RESUltados}

\subsection{Modelo de Precios para una variable exógena}

El Modelo de Insumo-Producto original desarrollado por Leontief se basó en unidades físicas (toneladas de grano, metros de tela, horashombre de fuerza laboral, entre otras); los coeficientes técnicos de 
insumos se sustentaron en cantidades físicas de insumos divididas entre cantidades físicas de productos. Posteriormente, esta información se estructuró como una tabla de intercambios (en un año base) en términos de valor, utilizando precios unitarios del año base.

Sobre ello, Leontief (1986) aseveró que todas las cifras de la tabla de valor, es este caso la MIP, resultan susceptibles de interpretarse como representaciones de cantidades físicas de los bienes o servicios generados por la economía a la que hace referencia; esto solo requiere que la unidad física de medida de las entradas se redefina como igual a la cantidad de producción del sector particular que puede comprarse por $\$ 1$ a precios de año base. Las matrices estructurales suele calcularse a partir de tablas de entrada-salida descritas en términos de valor; en cualquier caso, los coeficientes de entrada para fines analíticos deben interpretarse como relaciones de dos cantidades medidas en unidades físicas.

Como ya se mencionó, el Modelo de Precios sigue la metodología aplicada por Leontief en el análisis Insumo-Producto; en aras de la simplicidad en el registro, las interrelaciones monetarias se organizan con el supuesto de que todo el valor agregado se representa por la fuerza laboral. Además, se toman los valores de la MIP para los tres sectores, sin las filas correspondientes a fuerza laboral y producción, dado que simplemente se trata de la suma de los consumos intermedios de los tres sectores y la suma de las actividades industriales, importaciones e impuestos, respectivamente. Asimismo, se agrega a la tabla de la Demanda Intermedia la fuerza laboral como un solo insumo primario (incluyendo importaciones e impuestos) y se agregan los elementos de la demanda final. Como resultado, se determinan los precios del año base, tal como se muestra en el Cuadro 4.

Cuadro 4

PRECIOS EN MILLONES DE PESOS A PRECIOS BÁSICOS DE 2013

\begin{tabular}{cccccc}
\hline Actividades & $\mathbf{1}$ & $\mathbf{2}$ & $\mathbf{3}$ & $\mathbf{f}_{\mathbf{1}}$ & Producción total \\
\hline 1 & 64,515 & 421,732 & 5,170 & 288,325 & 779,742 \\
\hline 2 & 117,098 & $3,021,320$ & 746,758 & $9,753,926$ & $13,639,102$ \\
\hline 3 & 55,740 & $1,853,896$ & $1,805,456$ & $9,508,711$ & $13,223,804$ \\
\hline Fuerza laboral & 542,389 & $8,342,154$ & $10,666,420$ & $2,009,673$ & $21,560,636$ \\
\hline Producción & 779,742 & $13,639,102$ & $13,223,804$ & $21,560,636$ & \\
\hline
\end{tabular}

Fuente: Elaboración propia con base en la MIP de México a precios básicos de 2013 (INEGI, 2017). 
Se obtiene la matriz de coeficientes técnicos A del cuadro 4:

$$
\text { Matriz } A=\left[\begin{array}{llll}
0.0827 & 0.0309 & 0.0004 & 0.0134 \\
0.1502 & 0.2215 & 0.0565 & 0.4524 \\
0.0715 & 0.1359 & 0.1365 & 0.4410 \\
0.6956 & 0.6116 & 0.8066 & 0.0932
\end{array}\right]
$$

Por lo que la matriz transpuesta es:

$$
\text { Matriz } \boldsymbol{A}^{\prime}=\left[\begin{array}{cccc}
\mathbf{0 . 0 8 2 7} & \mathbf{0 . 1 5 0 2} & \mathbf{0 . 0 7 1 5} & 0.6956 \\
\mathbf{0 . 0 3 0 9} & \mathbf{0 . 2 2 1 5} & \mathbf{0 . 1 3 5 9} & 0.6116 \\
\mathbf{0 . 0 0 0 4} & \mathbf{0 . 0 5 6 5} & \mathbf{0 . 1 3 6 5} & 0.8066 \\
0.0134 & 0.4524 & 0.4410 & 0.0932
\end{array}\right]
$$

Ahora, $\left(\mathrm{L}^{0}\right)^{\prime}=\left(\mathrm{I}-\mathrm{A}^{\prime}\right)^{-1}$ para los tres sectores indicados con negritas en la matriz anterior; entonces:

$$
\left(\boldsymbol{L}^{\mathbf{0}}\right)^{\prime}=(\boldsymbol{I}-\boldsymbol{A})^{-1}=\left[\begin{array}{lll}
1.0977 & 0.2209 & 0.1256 \\
0.0442 & 1.3083 & 0.2096 \\
0.0034 & 0.0857 & 1.1719
\end{array}\right]
$$

De los datos del año base 2013:

$$
v_{c}^{0}=\left[\begin{array}{l}
0.6956 \\
0.6116 \\
0.8066
\end{array}\right]
$$

Por lo que:

$$
\tilde{p}^{0}=\left(L^{0}\right)^{\prime} v_{c}^{0}=\left[\begin{array}{lll}
1.0977 & 0.2209 & 0.1256 \\
0.0442 & 1.3083 & 0.2096 \\
0.0034 & 0.0857 & 1.1719
\end{array}\right]\left[\begin{array}{l}
0.6956 \\
0.6116 \\
0.8066
\end{array}\right]=\left[\begin{array}{l}
1.0000 \\
1.0000 \\
1.0000
\end{array}\right]
$$

Esto reproduce los precios índices del año base 2013, tal como se esperaba. La lógica reside en que los cambios en los precios de los insumos labores (o, más generalmente, los precios primarios de los insumos) llevan cambios en los costos unitarios sectoriales (y, por lo tanto, en los precios de producción y no en las cantidades de ésta). Por ejemplo, los aumentos de costos se transfieren completamente a medida que se eleva el precio de los insumos intermedios a todos los compradores, que a su vez los traspasan al incrementar sus precios de producción en consecuencia, etcétera. A diferencia del Modelo de Insumo-Producto de Leontief, conocido como el Modelo de Empuje, el Modelo de Precios es mejor conocido como el Modelo de Insumo-Producto de Empuje de Costos (Dietzenbacher, 1997), en el cual las cantidades se mantienen 
fijas y los precios se modifican de acuerdo con las condiciones presentes o con los supuestos considerados.

Debido a que las renegociaciones del TLCAN no han rendido frutos hasta el momento, para calcular el impacto de las importaciones de productos agropecuarios en los tres sectores de nuestro país, se consideran la imposición de aranceles al acero y al aluminio por Estados Unidos, así como la respuesta de México al proceder del miso modo respecto a los productos del sector agropecuario destinados al país del norte.

Así, suponiendo un incremento de precios en el sector agropecuario de un $20 \%$, de 0.6956 a 0.8347 , mientras que los precios de los sectores 2 y 3 permanecen sin cambios, el vector de los nuevos costos es: Por lo que:

$$
\boldsymbol{v}_{c}^{1}=\left[\begin{array}{c}
\mathbf{0 . 8 3 4 7} \\
0.6116 \\
0.8066
\end{array}\right]
$$

Sobre los precios del índice original, se tiene que el precio del sector 1

$$
\tilde{\boldsymbol{p}}^{\mathbf{0}}=\left(\boldsymbol{L}^{\mathbf{0}}\right)^{\prime} \boldsymbol{v}_{\boldsymbol{c}}^{1}=\left[\begin{array}{lll}
1.0977 & 0.2209 & 0.1256 \\
0.0442 & 1.3083 & 0.2096 \\
0.0034 & 0.0857 & 1.1719
\end{array}\right]\left[\begin{array}{c}
\mathbf{0 . 8 3 4 7} \\
0.6116 \\
0.8066
\end{array}\right]=\left[\begin{array}{l}
1.1527 \\
1.0061 \\
1.0004
\end{array}\right]
$$

se incrementó a 1.1527 (15.27\%); el del sector 2, a 1.0061 (0.61\%); y el del 3, a $1.0004(0.04 \%)$.

\section{CONCLUSIONES}

A raíz de la estrategia del gobierno de nuestro país para responder a los aranceles impuestos por Estados Unidos al acero y aluminio, se presenta la estimación del efecto multiplicador en los tres grandes sectores de la economía a partir del agregado de aranceles a productos agropecuarios que importa México de Estados Unidos (que van de 15\% hasta 25\% e incluyen manzanas, productos lácteos, bovinos y porcinos), a través de escenarios de simulación de incremento de precios promedio y de la aplicación de la Teoría de Análisis Estructural. 
Se encuentra que el principal resultado se produce en el mismo sector agropecuario con un efecto directo de $15.27 \%$, e indirecto en aumento de precios en los sectores secundario $(0.61 \%)$ y terciario (0.04\%). Por tanto, la imposición de aranceles perjudica inmediatamente a los productores de Estados Unidos, quienes tendrán que vender a un precio más alto, lo que representa un problema para el consumidor doméstico (en México) pues, al incrementar los precios de compra, disminuye el nivel del poder adquisitivo.

El gobierno mexicano debe considerar los efectos generados en la economía nacional por la imposición de aranceles a productos de consumo doméstico y, en consecuencia, cambiar su estrategia de negociación para que de inmediato se retomen las negociaciones del TLCAN, con la toma de acuerdos que convengan a los tres involucrados como prioridad.

\section{Bibliografía}

Appendini, K. (2018), “Informes Taller TLCAN/UE”, Estudios Demográficos y Urbanos, 33 (3), pp. 805-815.

Burgos, K. (2007), Análisis de multiplicadores y elasticidades para la economía chilena base 2003, mediante un enfoque input-output, Universidad del BioBio.

Camargo, F. (2017), “Dinámica de la producción regional en México", Paradigma económico, (2), pp. 127-166.

Carbajal, Y. y Del Moral, L. (2014), "El desempeño del sector automotriz en México en la era TLCAN. Un análisis a 20 años", Paradigma económico, (2), pp. 95-126.

Celis, D. (2018), "Ascendieron las exportaciones en México en el sector agroalimentario en 2017", en Asociación Nacional de Tiendas de Autoservicio y Departamentales, disponible en: https://antad.net/ascendieron-las-exportaciones-en-mexico-en-el-sector-agroalimentario-en-2017/.

Chávez, A. (2017), Análisis multisectorial del incremento en las gasolinas y el diesel en la economía mexicana, El Colegio de México, México.

Chraki, F. B. (2016), "Análisis input-output de encadenamientos productivos y sectores clave en la economía", Finanzas Políticas Económicas, 8 (1), pp. 55-81.

Cortés, M. (2018), “TLCAN: negociaciones bilaterales, acuerdo trilateral”, Dinero en Imagen, disponible en: https://www.dineroenimagen.com/maricarmen-cortes/tlcan-negociaciones-bilaterales-acuerdo-trilateral/101797.

Dietzenbacher, E. (1997), "In Vindication of the Gosh Model: A Reinterpretation as a Price Model", Journal of Regional Science, (37), pp. 629-651. 
DOF (2018). Diario Oficial de la Federación.

Figueroa, C. y Obed, C. (2015), "Identificación de los sectores clave de la economía mexicana", Investigación y Ciencia, 23 (65), pp. 48-58.

Fuentes, N. (2005), "Construcción de una matriz regional de insumo-producto", Problemas del Desarrollo, (36).

Gazcón, F. (2017), "Expertos descartan catástrofe sin TLC; reglas de la OMC entrarían en vigor", Excélsior, 12 febrero, disponible en: https://www.excelsior.com.mx/nacional/2017/02/12/1145806.

Hernández, G. (2012), “Matrices insumo-producto y análisis de multiplicadores: una aplicación para Colombia”, Revista de Economia Institucional, 14 (26), pp. 203-221.

INEGI (2017), "INEGI- PIB y cuentas nacionales, matriz de insumo-producto", INEGI, disponible en: http://www.inegi.org.mx/est/contenidos/proyectos/ $\mathrm{cn} / \mathrm{mip} 13 /$ default.aspx $\% 0 \mathrm{~A}$.

Leontief, W. (1986), Input-Output Economics, Oxford University Press, Nueva York.

Leontief, W. (1993), Análisis económico input-output, Printer Industria Gráfica, México.

Miller, R. y Blair, P. (2009), Input-Output Analysis Foundations and Extensions, Cambrigde, doi: 10.1007/s13398-014-0173-7.2.

Núñez, G. y J. Romero (2016), “Contabilidad insumo-producto y un análisis comparativo-estructural de la economía mexicana" Serie de documentos de trabajo del Centro de Estudios Económicos 2016-5, El Colegio de México. México, D.F.

Oosterhaven, J. (1996), "Leontief versus Ghoshian Price and Quantity Models", Southern Economic Journal, 62 (3), pp. 750-759, doi: 10.2307/1060892.

Ramos, C. et al. (2013), "Un análisis de la descomposición de la rama eléctrica en las tablas input-output", en Evolución y contrastes de las metodologías sobre la relación economía-industria y empleo, Universidad de Deusto, Donostia-San Sebastián, pp. 53-68.

Ramos, F. (2017), "Posibles escenarios del sector agropecuario ante la cancelación del TLCAN", en Hortalizas, disponible en: https://www.hortalizas. com/poscosecha-y-mercados/posibles-escenarios-del-sector-agropecuarioante-la-cancelacion-del-tlcan/.

Urrutia, M. et al. (2017), “Contribución del sector pecuario a la economía mexicana. Un análisis desde la Matriz Insumo Producto", Revista Mexicana de Ciencias Pecuarias, 8 (1), pp. 31-41, doi: 10.22319/rmcp.v8i1.4308.

Yúnez, A. y Barceinas, F. (2004), "The agriculture of Mexico after ten years of NAFTA implementation", en Documentos de Trabajo, Banco Central de Chile, (277), p. 1.

Yúnez, A. y González, S. (2008), "Efectos multiplicadores de las actividades productivas en el ingreso rural en México", El Trimestre Económico, 75 (298), pp. 349-377. 